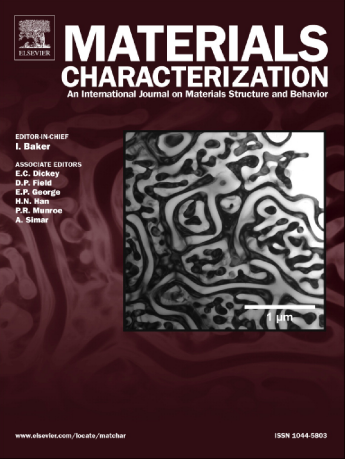

Identification of colloidal silica polishing induced contamination in silicon

D. Tweddle, P. Hamer, Z. Shen, M.P. Moody, P.R. Wilshaw

\author{
S1044-5803(19)30679-5 \\ https://doi.org/10.1016/j.matchar.2019.04.029 \\ MTL 9698
}

DOI:

Reference:

To appear in:

Materials Characterization

Received date:

5 March 2019

Revised date:

23 April 2019

Accepted date:

23 April 2019

Please cite this article as: D. Tweddle, P. Hamer, Z. Shen, et al., Identification of colloidal silica polishing induced contamination in silicon, Materials Characterization, https://doi.org/10.1016/j.matchar.2019.04.029

This is a PDF file of an unedited manuscript that has been accepted for publication. As a service to our customers we are providing this early version of the manuscript. The manuscript will undergo copyediting, typesetting, and review of the resulting proof before it is published in its final form. Please note that during the production process errors may be discovered which could affect the content, and all legal disclaimers that apply to the journal pertain. 


\title{
Identification of Colloidal Silica Polishing Induced Contamination in Silicon
}

\author{
D. Tweddle ${ }^{1 *}$, P. Hamer ${ }^{2}$, Z. Shen ${ }^{1}$, M. P. Moody ${ }^{1}$, P. R. Wilshaw ${ }^{1}$. \\ ${ }^{1}$ University of Oxford, Department of Materials, 16 Parks Road, Oxford, OX1 3PH, UK \\ ${ }^{2}$ University of New South Wales, School Photovoltaic and Renewable Energy Engineering, Sydney \\ 2052, Australia
}

*Corresponding author: david.tweddle@ materials.ox.ac.uk

Keywords: Silicon; Polishing; Contamination; Atom Probe Tomography; Correlative Microscopy.

\begin{abstract}
This paper presents a multiscale characterisation approach, analysing the effect of colloidal silica polishing on crystallographic defects in multicrystalline silicon. Colloidal silica polishing for as little time as 30 minutes was found to significantly increase the recombination activity of all defects, as measured by Electron Beam Induced Current Mapping. The impurities responsible for the room temperature contamination of defects due to colloidal silica polishing were $\mathrm{Cu}$ and $\mathrm{Ni}$, as measured by Atom Probe Tomography.
\end{abstract}

\section{Introduction}

A number of recent studies have used combinations of electron beam induced current (EBIC) mapping, electron backscatter diffraction (EBSD) and atom probe tomography (APT) to analyse impurities present at electrically characterised crystallographic defects in silicon [1], [2]. In the first stages of specimen preparation, prior to the application of any of these experimental techniques, a sample with a flat surface of lower than $50 \mathrm{~nm}$ in roughness is preferred [3]. A conventional method for producing such a surface is chemomechanical polishing using colloidal silica, which has been used extensively in a number of material systems [4]-[6], dependent upon the purity of the colloidal silica used. However, this technique has been historically linked with contamination in silicon [7]-[9]. With the increased interest in advanced microscopy of silicon for photovoltaic applications, it is imperative to ensure sample preparation does not introduce contamination prior to analysis.

Transition metals are notorious impurities in multicrystalline silicon and are known to significantly increase carrier recombination via the introduction of deep levels into the band gap [10]. These impurities have been found to segregate to crystallographic defects, such as grain boundaries and dislocations [11], in addition to forming large intragranular metallic precipitates [12]. Nickel and copper are both known to limit the performance of multicrystalline silicon solar cells, by the decoration of crystallographic defects [13], [14]. They are of particular interest to solar cell producers as potential contaminants, due to their high diffusivity and solubility in silicon enabling the potential for significant in-diffusion at very low temperatures, indeed even room temperature.

Nickel is easily introduced from contamination of the wafer surface at elevated temperatures [15]. Since Ni diffuses interstitially and is neutral at any position of the Fermi level in the band gap [15], its diffusivity has been the subject of much debate. The most commonly used diffusivity is $\mathrm{D}_{\mathrm{Ni}}(\mathrm{T})=2.3 \mathrm{x}$ $10^{-3} \exp \left(-0.47 \mathrm{eV} / \mathrm{k}_{\mathrm{B}} \mathrm{T}\right) \mathrm{cm}^{2} / \mathrm{s}$, as determined from neutron activation analysis (NAA) [16]. However, this value has been the subject of much scrutiny of late due to the activation energy differing by more than a factor of two compared to the value of $0.21 \mathrm{eV}$ calculated by the nudged elastic band (NEB) method [17]. Further, a more recent study, using an Al-Si sink layer to immobilise Ni during and after cooling, produced a new description of diffusivity with $\mathrm{D}_{\mathrm{Ni}}(\mathrm{T})=1.69 \times 10^{-4} \exp \left(-0.15 \mathrm{eV} / \mathrm{k}_{\mathrm{B}} \mathrm{T}\right) \mathrm{cm}^{2} / \mathrm{s}$ which is applicable over the temperature range $665-885^{\circ} \mathrm{C}$ [15]. 
Correspondingly, the high diffusivity of copper allows it to be easily introduced by contamination of the wafer surface at elevated temperatures [18]. Unlike most other $3 \mathrm{~d}$ transition metals, Cu diffuses in silicon in the $\mathrm{Cu}^{+}$charge state [19]. The first direct experimental measurements of the diffusivity of copper at room temperature ( 240 - $380 \mathrm{~K}$ range), were performed by Istratov using the transient ion drift technique in low boron-doped float zone silicon [20]. An expression for the interstitial $\mathrm{Cu}$ intrinsic diffusion coefficient was given as $\mathrm{D}_{\mathrm{Cu}}(\mathrm{T})=\left(3.0 \times 10^{-4}\right) \exp \left(-0.18 \mathrm{eV} / \mathrm{k}_{\mathrm{B}} \mathrm{T}\right) \mathrm{cm}^{2} / \mathrm{s}$.

Nickel and copper have the highest solubilities of the $3 \mathrm{~d}$ transition metals in silicon. The solubility for $\mathrm{Ni}$ and $\mathrm{Cu}$ are given as the equilibrium concentration in silicon as a function of temperature [Ni] = $\exp \left(3.2-\left(1.68 \mathrm{eV} / \mathrm{k}_{\mathrm{B}} \mathrm{T}\right)\right)$ and $[\mathrm{Cu}]=\exp \left(2.4-\left(1.49 \mathrm{eV} / \mathrm{k}_{\mathrm{B}} \mathrm{T}\right)\right)$ [21]. Although these expressions were determined at much greater temperatures, it is possible by extrapolation, to estimate the room temperature solubility concentrations. These are much lower than the levels determined by Coletti to cause significant amounts of bulk recombination in silicon [22].

The low energy barrier to diffusion and high diffusivity even at low temperatures, make $\mathrm{Cu}$ and $\mathrm{Ni}$ prime candidates for room temperature contamination. Indeed, evidence for room temperature diffusion of copper was found historically by Prigge et al. [7] and Wagner et al. [8], who confirmed that $\mathrm{Cu}$ can be introduced colloidal silica polishing, and increase the recombination activity of the wafer. Room temperature diffusion of Ni was also found by Yarykin and Weber [23], investigating wet chemical etching in contaminated alkaline solutions. By identifying the deep-level response from the electrically active NiVO defect, a lower limit for the diffusivity at $35{ }^{\circ} \mathrm{C}$ was determined as $10^{-9}$ $\mathrm{cm}^{2} / \mathrm{s}$.

Since both copper and nickel have been found at grain boundaries in APT [24], [25], it is important that methods are developed that can identify unintentional contamination being introduced during sample preparation prior to analysis. In this paper, a correlative EBIC, EBSD and APT method is demonstrated in order to understand the effect of colloidal silica polishing on the electrical properties of defects in multicrystalline silicon and to show that both copper and nickel decoration of crystallographic defects can be produced by colloidal silica polishing at room temperature.

\section{Experimental Methods}

The materials used in this study are adjacent, as-sawn, sister wafers taken from a high performance ptype multicrystalline silicon ingot. The p-type wafers have a resistivity of $0.3 \Omega . \mathrm{cm}$ and a corresponding boron doping concentration of $6 \times 10^{16} \mathrm{~cm}^{-3}$. Diamond suspension polishing using $0.25 \mu \mathrm{m}$ particles was performed to ensure a flat surface and then any remaining saw or polishing damage was removed using a chemical etch of hydrofluoric acid $(50 \mathrm{ml})$, nitric acid $(220 \mathrm{ml})$ and acetic acid $(30 \mathrm{ml})$. EBIC was then performed on the wafer, followed by a 12 hour polish, using Kemet Colloidal Silica (Col-k) under standard laboratory conditions, with wafers attached to a borosilicate glass plate to prevent contamination from the holder. EBIC characterisation was then repeated. In order to investigate whether measurable contamination can still occur in shorter time frames, a separate wafer was polished using colloidal silica for 30 minutes and analysed before and after using EBIC.

Prior to EBIC, a Schottky contact was formed on the surface, consisting of a $13 \mathrm{~nm} \mathrm{Al}$ thermally evaporated layer. EBIC was performed in a Zeiss NVision FIB/SEM with an accelerating voltage of $12.5 \mathrm{kV}$ using an Oxford Instruments EBIC system comprising an Omniprobe 200 surface probe connected to a Point Electronic amplifier. After the removal of the surface Al Schottky barrier, EBSD was performed using a Zeiss Crossbeam 540 equipped with an Oxford Instruments EBSD Nordlys Max 3 detector, at an accelerating voltage of $30 \mathrm{kV}$ and beam current of $3 \mathrm{nA}$. Atom probe needles incorporating a targeted grain boundary in each of the wafers, respectively, were then produced using the method described in [26]. This method allows for the production of needles containing a grain boundary running along the length of the specimen, enabling a comparatively large section of the 
boundary to be analysed. APT was performed using a LEAP $5000 \mathrm{XR}$, at a temperature of $50 \mathrm{~K}$, detection rate of $1.5 \%$, laser energy of $50 \mathrm{pJ}$ and pulse frequency of $200 \mathrm{kHz}$.

\section{Results and Analysis}

In order to investigate the effect of colloidal silica polishing on the electrical properties, EBIC measurements were made on the same wafer before and after colloidal silica polishing. EBIC images are shown in Figure 1, with the numerical value of contrast of selected grain boundaries indicated in the higher magnification images $\mathrm{c}$ and $\mathrm{d}$. It is clear that the grain boundaries become significantly more electrically active after colloidal silica polishing. Interestingly, not all grain boundaries respond equally to the polishing process, with some grain boundaries becoming significantly more recombination active than others. In addition, intragrain dislocations, which are not observed in the EBIC image before polishing, become active and visible after colloidal silica polishing. This is a clear indication that impurities have been introduced by the polishing process and have segregated to crystallographic defects, introducing recombination active levels into the band gap. 
a)

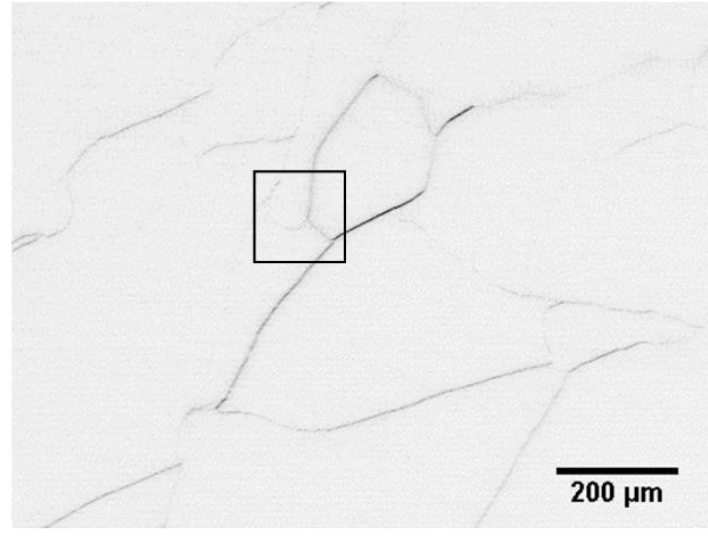

c)

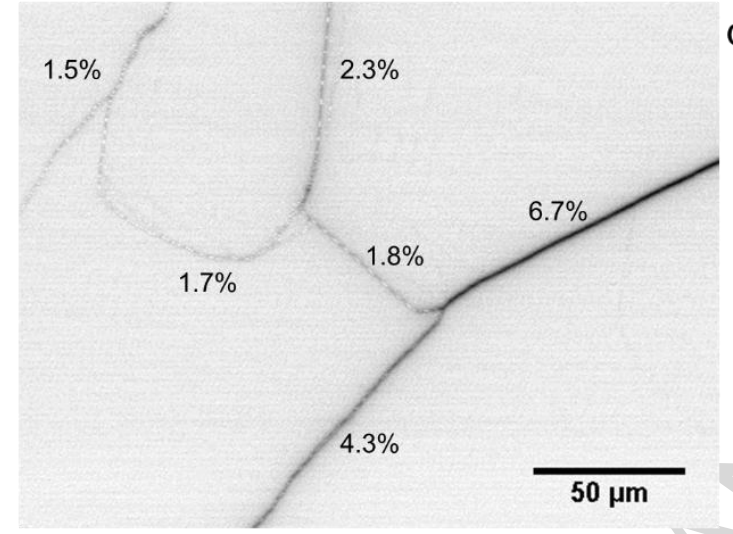

e)

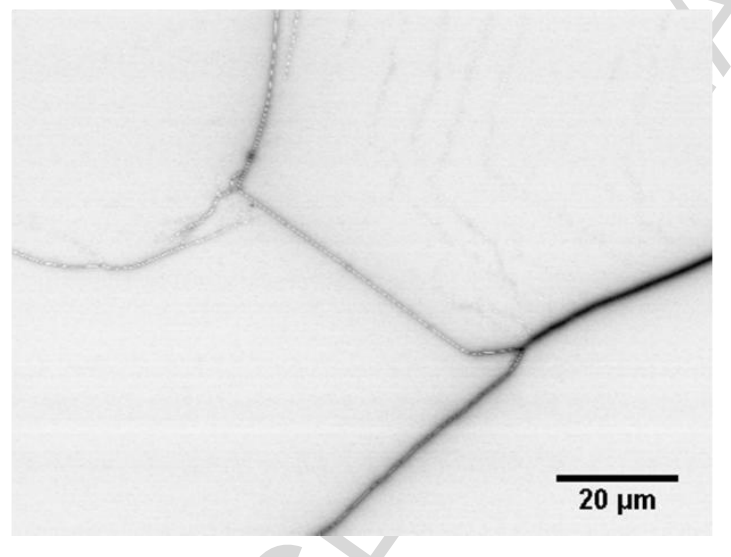

b)

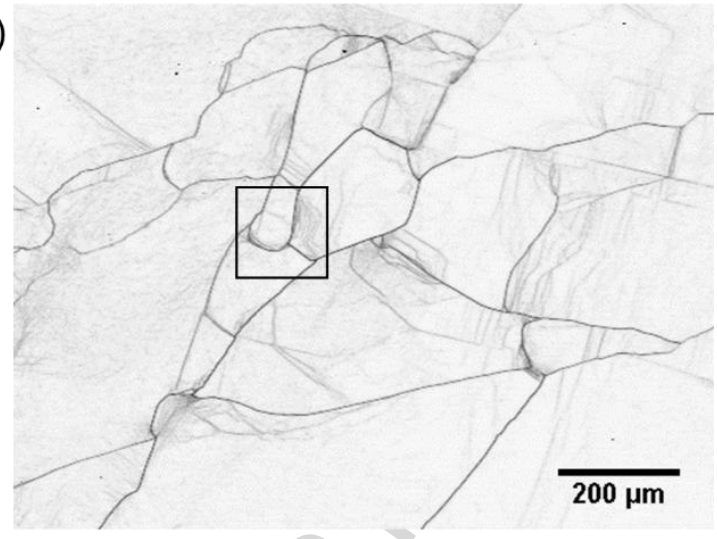

d)

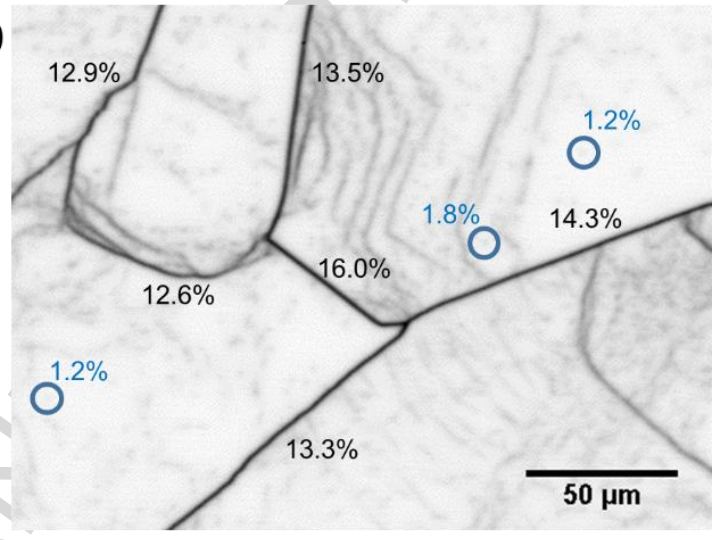

f)

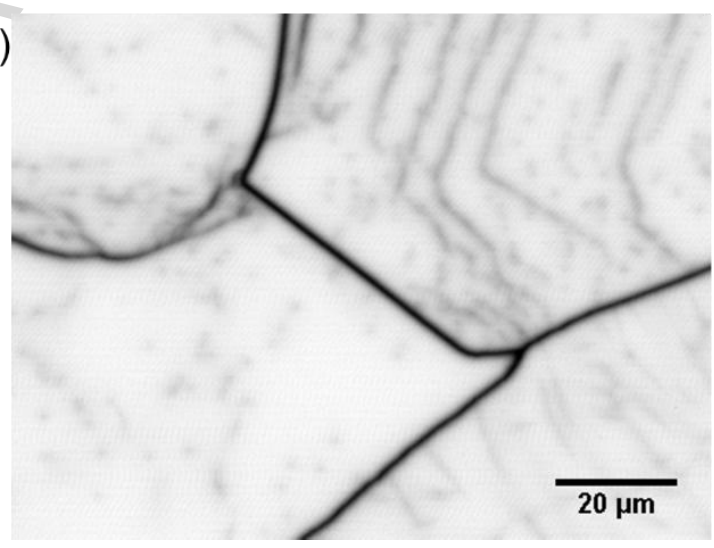

Figure 1. Electron beam induced current images of the same wafer before and after colloidal silica polishing, with a) being prior to colloidal silica and $b$ ) subsequent to colloidal silica polishing. $c$ ) and d) are magnified EBIC images of a) and $b$ ) respectively, from the region of interest marked. In c) and d) the EBIC contrast of the grain boundaries before and after colloidal silica polishing, and for three dislocations not previously observed before polishing, are displayed. e) and f) show higher magnification EBIC images before and after colloidal silica polishing respectively.

These boundaries were confirmed as small angle grain boundaries using EBSD (Figure 2a). Interestingly, before polishing, the more recombination active grain boundaries were found to have the greatest misorientation (Figure $2 b$ ). This corresponds to a higher dislocation density along the grain boundary and therefore more sites for impurities [27]. After colloidal silica polishing this trend becomes less evident, with a notable outlier at a misorientation of $2^{\circ}$, a general increase in the EBIC contrast for greater grain boundary misorientations was observed. 
a)

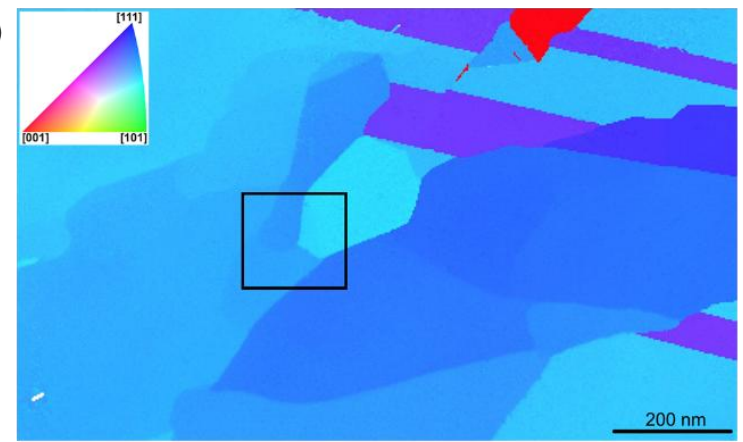

b)

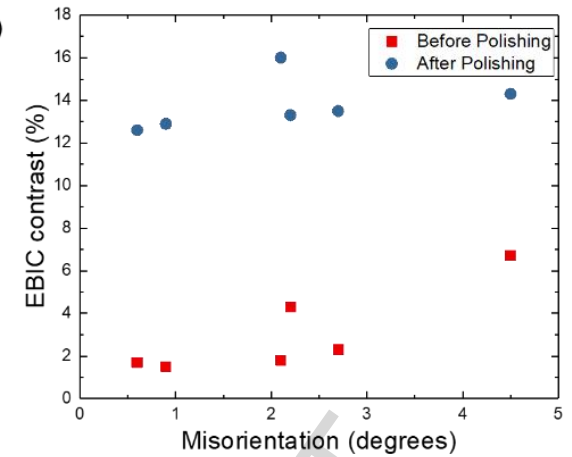

Figure 2. a) EBSD inverse pole figure (IPFY) map of sample measured in Figure 1, with region selected for EBIC at greater magnification marked. b) EBIC contrast for grain boundaries marked in Figure 1 plotted against grain boundary misorientation.

In order to determine which impurities are introduced from the colloidal silica polishing process and the extent of this contamination, APT of an identical grain boundary in sister wafers with and without a 12 hour colloidal silica polish was performed. This boundary was a random angle grain boundary with a misorientation of $44^{\circ}$ and no evidence of dislocations. This specimen was taken from a different wafer than that shown in Figure $1+2$. Figure 3 shows the mass spectra of the two grain boundaries. The mass spectrum of the polished sample shows distinctive peaks for $\mathrm{Ni}$ and $\mathrm{Cu}$, not seen in the unpolished sample. For the unpolished grain boundary sample, the only peak observed in the mass region where the $\mathrm{Cu}$ and $\mathrm{Ni}$ peaks are observed is at $60 \mathrm{Da}$, which coincides with $\mathrm{SiO}_{2}{ }^{+}$, with no detectable level of nickel or copper. This indicates that significant quantities of $\mathrm{Ni}$ and $\mathrm{Cu}$ were introduced as a result of the polishing process.
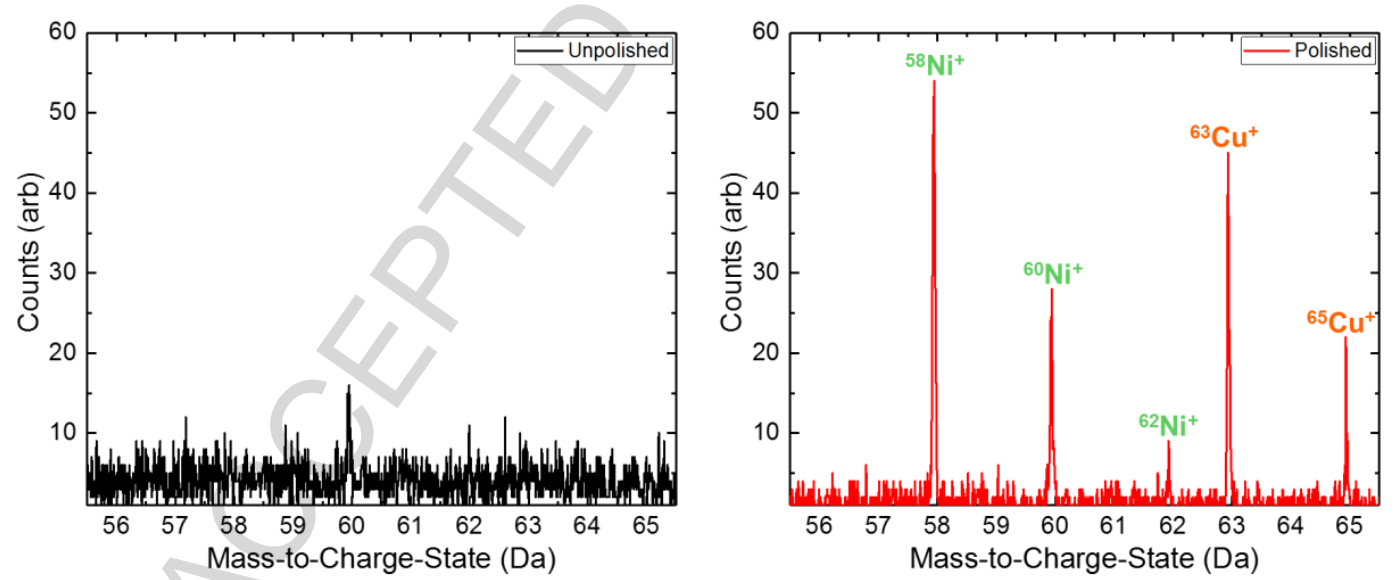

Figure 3. Mass spectra of two identical grain boundaries, taken from sister wafers, which have been left unpolished and been exposed to colloidal silica polishing for a period of 12 hours. In the polished sample, the nickel and copper peaks are labelled.

The copper and nickel found in the mass spectrum of the polished sample, are shown to segregate to the grain boundary in the APT atom maps in Figure 4. The grain boundary was also enriched in both carbon and oxygen (not shown), as seen previously [14]. It is worth stating that counts of $\mathrm{Ni}$ and $\mathrm{Cu}$ found away from the boundary are background noise in the dataset. 
a)

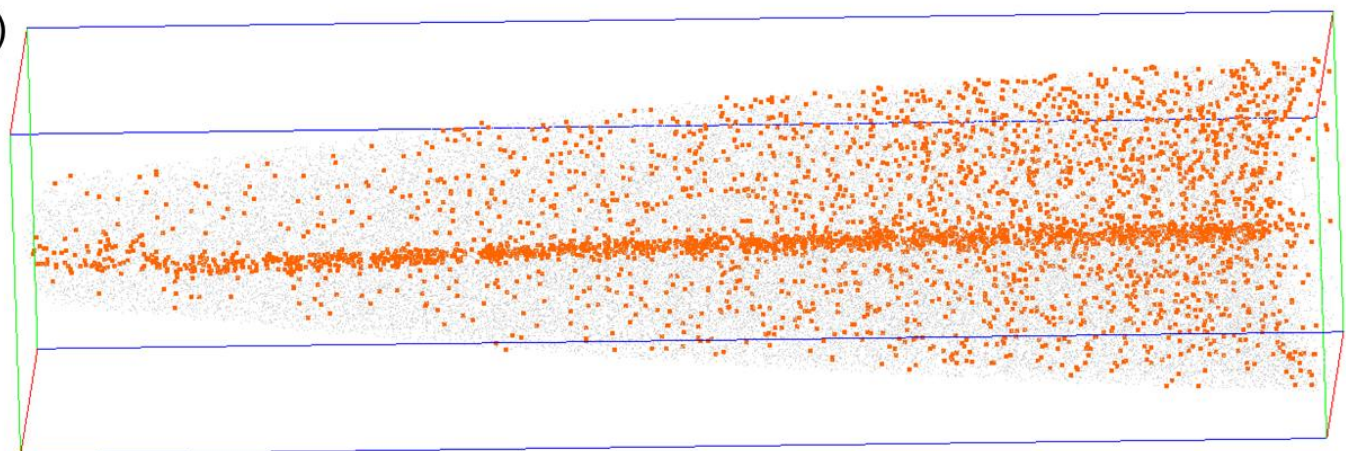

$50 \mathrm{~nm}$

b)

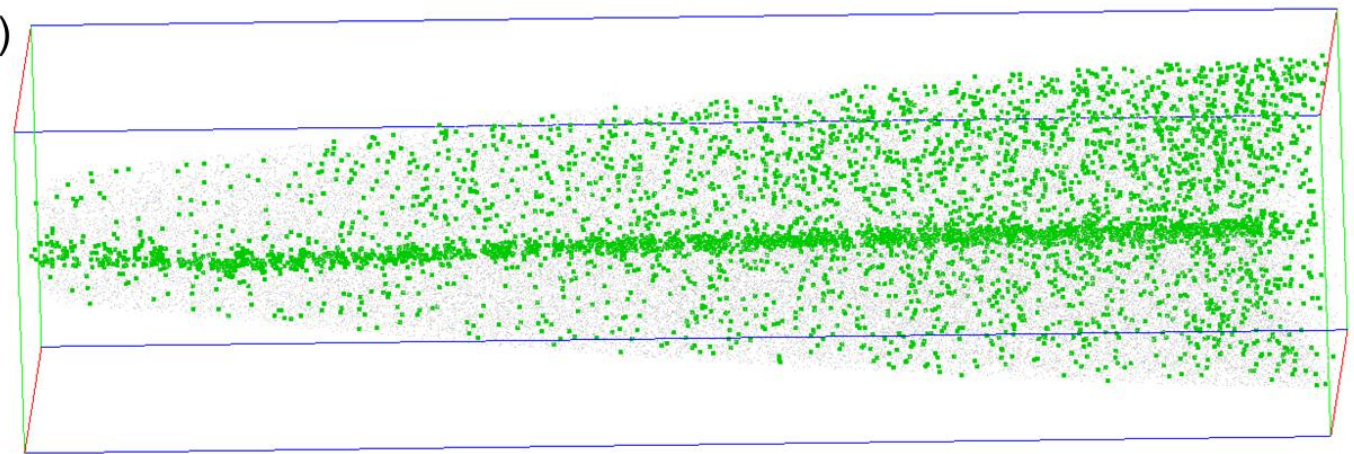

Figure 4. Atom probe reconstruction containing a grain boundary from the sample which had undergone a 12 hour polish in colloidal silica. A significant excess of a) copper and b) nickel are observed at the boundary.

EBIC and APT analysis shown above, were performed on wafers which had been exposed to an extensive 12 hour polishing process. However, in the literature, colloidal silica polishing is often only performed for much shorter times. To investigate whether measurable contamination can occur in these times, a 30 minute colloidal silica polish was performed on a separate wafer, with EBIC measurements made before and after (Figure 5). From Figure 5, it is clear that polishing significantly increases the contrast of all grain boundaries measured. Additionally, previously unmeasurable intragranular dislocations become electrically active after polishing. By comparing EBSD and EBIC, the increase in recombination activity with greater misorientation, observed in Figure 2, is less clear, with the lowest EBIC contrast after polishing observed at the greatest misorientation. 


\section{Before Polishing}

a)

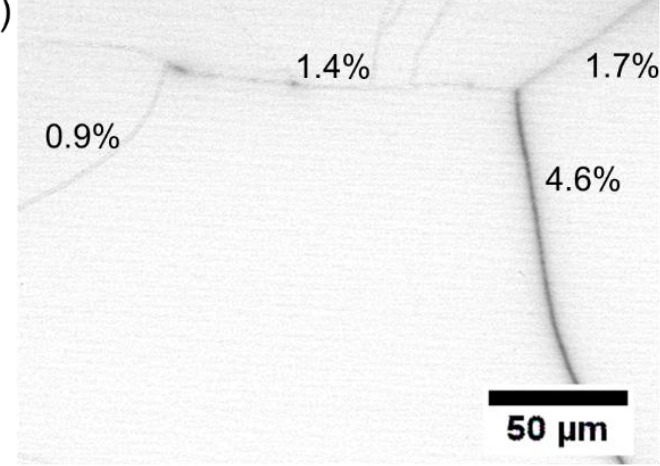

b)

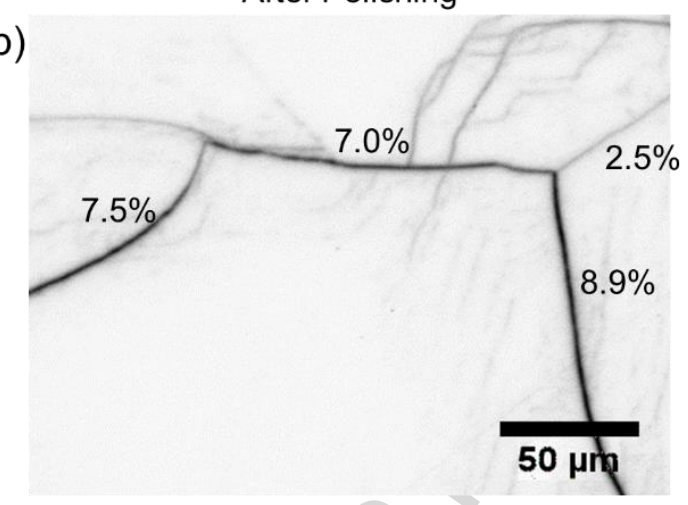

After Polishing

c)
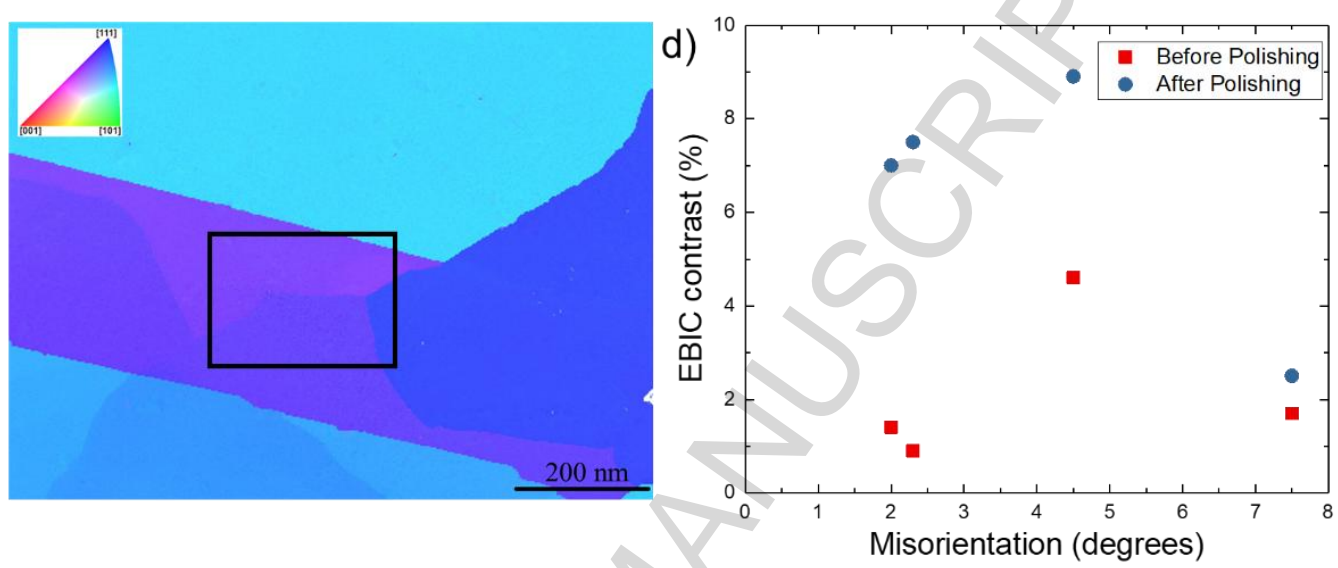

Figure 5. Electron beam induced current images of the same wafer before a) and after b) a 30 minute colloidal silica polish. EBIC contrast for a number of grain boundaries are displayed on the figure. $c$ ) EBSD inverse pole figure (IPFY) map, with region selected for EBIC at greater magnification marked. d) EBIC contrast for grain boundaries marked plotted against grain boundary misorientation.

\section{Discussion}

Unintentional incorporation of metallic impurities during the various processing stages of the silicon wafer is a significant issue in the silicon photovoltaics community, due to the very low base levels of impurities and high diffusivity of some transition metals. Nickel and copper have both been identified as potential contaminants, which significantly hinder the efficiency of solar cells [22].

Here, the detrimental effect of colloidal silica polishing, to produce a flat surface prior to microscopical analysis, was investigated. The effect of colloidal silica on the electrical properties was examined using EBIC mapping (Figure 1). After polishing, all of the grain boundaries measured became much more electrically active. In addition, intragrain dislocations appear recombination active after polishing. Since it is known that clean dislocations are essentially electrically inactive [28], the activation after polishing indicates decoration by impurities in the polishing solution. This phenomenon was previously noticed by Kittler and Seifert [29], who commented on the 'peculiar' influence of polishing on (EBIC) contrast. They noted that dislocations, which were previously inactive became electrically active at room temperature. However, no reference to contamination was given, since no direct evidence of contamination at defects was achievable at that time.

The source of this contamination during the colloidal silica polishing process is unknown, although it is well known that such polishing can contain and introduce significant levels of impurities [30], [31]. An additional source of contamination, is from the distilled water used to clean the samples and also the polishing pad itself. A notable factor, which may contribute significantly to the levels of contamination observed, is the high $\mathrm{pH}$ of the polishing solution. In general, basic solutions are used 
to remove organic contaminants and acidic solutions are more effective at removing heavy metallic contamination [32]. It is for this reason, in the solar cell community, chemomechanical polishing slurry chemistries have been optimised to limit contamination [4]. It should be noted that in this work a standard colloidal silica (Kemet Col-K) was used under normal laboratory conditions, with plenty of opportunities for contamination to occur. However, it is believed to be common practice in university laboratories for EBIC specimens to be made without recourse to carefully controlled clean room environments and thus the findings of this research, that colloidal silica polishing can introduce electrically active impurities at room temperature, are relevant to much other EBIC work in the literature.

The grain boundaries analysed by EBSD were found to be primarily small angle grain boundaries (Figure 2). These boundaries are often found to be significantly recombination active, and so appreciably reduce the efficiency of multicrystalline silicon solar cells [33]. In the work of Wang et al. [27], small angle grain boundaries can be well described by a collection of Lomer dislocations, with increasing dislocation densities at greater misorientations. The increased dislocation density at greater misorientations results in a larger number of sites for impurities and hence increased grain boundary recombination [34]. A notable outlier to this trend is seen in Figure 5d, where the grain boundary with greatest misorientation also had the lowest EBIC contrast after polishing. This replicates the work by Sameshima [35], who found that misorientation was not the sole indicator of recombination activity.

In order to determine the cause of the resultant activation of defects, APT of a random angle grain boundary before and after polishing, in sister wafers was performed. This technique provides both compositional and spatial information of impurities at individual crystallographic defects. By comparing the APT mass spectra of the grain boundary before and after colloidal silica polishing (Figure 3), it is clear that polishing introduced significant quantities of both $\mathrm{Cu}$ and $\mathrm{Ni}$, which segregated to the defect, resulting in their electrical activation [36].

Since this study analysed HPMC-Si, which has a relatively high quantity of grain boundaries, it is interesting to note the effect of such defects. It is well known that impurities segregate to crystallographic defects, regions of local disorder, in order to relieve strain caused by lattice mismatch [11]. Since the EBIC contrast of grain boundaries and intragranular dislocations becomes much greater after polishing, it is evident that $\mathrm{Cu}$ and $\mathrm{Ni}$ diffuse to and segregate at these defects at room temperature. However, since $\mathrm{Cu}$ and $\mathrm{Ni}$ are found co-located at the grain boundary, it is not possible to distinguish how much of a role each impurity plays in the increase grain boundary recombination activity.

$\mathrm{Cu}$ and $\mathrm{Ni}$ have a well-known affinity to grain boundaries in silicon, with previous work by Rizk using transmission electron microscopy, clearly confirming the preferential formation of nanosize $\mathrm{Cu}$ and Ni precipitates at the grain boundary [37]. Additional work by Ohno, using APT, co-segregated $\mathrm{Ni}$ and $\mathrm{Cu}$ precipitates were observed at specific grain boundary sites [25]. In this study, both $\mathrm{Cu}$ and $\mathrm{Ni}$ were observed to decorate the grain boundary, but no evidence of large precipitates was seen.

From EBIC measurements, it is clear that not all grain boundaries respond equally to colloidal silica polishing. Indeed, certain grain boundaries which had very low recombination activity prior to polishing become significantly more recombination active. We speculate this relates to the presence of large concentrations of grain boundary precipitation sites which are not previously occupied. This is analogous to the internal gettering and activation of previously undecorated random angle grain boundaries during phosphorus diffusion gettering [38].

Although APT analysis was performed on wafers exposed to a 12 hour colloidal silica polishing process, results shown in Figure 5 clearly show that polishing for just 30 minutes resulted in a significant increase in defect recombination activity. The average increase in EBIC contrast of 4 times 
for the 30 minute colloidal silica polished sample compares to an average increase of 6 times for the 12 hour polished sample. Considering that this sample was polished for over 24 times longer, we can presume two possibilities. First, impurities reach the defects much more quickly to begin with and then the concentration starts to plateau at the defects. The second possibility is that the electrical activity does not respond linearly to the impurity concentration. Indeed the work by Fell [39] concludes that the recombination activity responds linearly to the impurity concentration up to the point at which the Fermi level becomes pinned to the defect states. Atom probe tomography is a powerful tool that, in correlation with EBIC, could answer this question.

\section{Conclusions}

In this study, the effect of colloidal silica polishing, under standard laboratory conditions, on high performance multicrystalline silicon was determined. From the use of EBIC on the same wafer, before and after colloidal silica polishing, a large increase in recombination activity at both grain boundaries and previously inactive intragranular dislocations is revealed. It is therefore concluded that using colloidal silicon polishing of silicon containing defects can introduce copper and nickel decoration of those defects - even at room temperature. Such polishing should be avoided if meaningful conclusions are to be drawn regarding the electrical activity of different defects in silicon. Instead satisfactory specimens may be produced by a combination of diamond polishing followed by wet chemical etching.

\section{Acknowledgements}

The authors would like to acknowledge financial support from the UK government through the EPSRC (Supersilicon grant, EP/M024911/1). In addition, the Zeiss Crossbeam FIB/SEM used in this work was supported by EPSRC through the Strategic Equipment Fund, grant EP/N010868/1. P. Hamer would like to acknowledge support from the Australian Renewable Energy Agency (ARENA) through the Australian Centre for Advanced Photovoltaics (ACAP) and their fellowship program.

\section{References}

[1] A. Stoffers et al., "Correlating Atom Probe Tomography with Atomic-Resolved Scanning Transmission Electron Microscopy: Example of Segregation at Silicon Grain Boundaries," Microsc. Microanal., pp. 1-9, 2017.

[2] Y. Ohno et al., "Three-dimensional evaluation of gettering ability of sigma $3\{111\}$ grain boundaries in silicon by atom probe tomography combined with transmission electron microscopy," Appl. Phys. Lett., vol. 103, no. 10, pp. 12-16, 2013.

[3] B. AlMangour and J. M. Yang, "Improving the surface quality and mechanical properties by shot-peening of 17-4 stainless steel fabricated by additive manufacturing," Mater. Des., vol. 110, pp. 914-924, 2016.

[4] G. Fisher, M. R. Seacrist, and R. W. Standley, "Silicon crystal growth and wafer technologies," Proc. IEEE, vol. 100, no. SPL CONTENT, pp. 1454-1474, 2012.

[5] H. Aida, H. Takeda, K. Koyama, H. Katakura, K. Sunakawa, and T. Doi, "Chemical Mechanical Polishing of Gallium Nitride with Colloidal Silica," J. Electrochem. Soc., vol. 158, no. 12, p. H1206, 2011.

[6] Y. Ahn, J. Y. Yoon, C. W. Baek, and Y. K. Kim, "Chemical mechanical polishing by colloidal silica-based slurry for micro-scratch reduction," Wear, vol. 257, no. 7-8, pp. 785-789, 2004.

[7] H. Prigge, P. Gerlach, P. O. Hahn, A. Schnegg, and H. Jacob, "Acceptor Compensation in Silicon Induced by Chemomechanical Polishing," Electrochem. Soc., vol. 138, no. 5, pp. 1385-1389, 1991. 
[8] P. Wagner, H. Hage, H. Prigge, T. Prescha, and J. Weber, "Properties of copper-induced complexes in silicon," in roceedings of the Sixth International Symposium on Silicon Materials Science and Technology, 1990, pp. 675-686.

[9] P. F. Schmidt, "A Neutron Activation Analysis Study of the Sources of Transition Group Metal Contamination in the Silicon Device Manufacturing Process," J. Electrochem. Soc., vol. 128, no. 3, p. 630, 1981.

[10] A. Cuevas, M. Stocks, D. Macdonald, M. Kerr, and C. Samundsett, "Recombination and trapping in multicrystalline silicon," IEEE Trans. Electron Devices, vol. 46, no. 10, pp. 2026 2034, 1999.

[11] J. Chen, T. Sekiguchi, D. Yang, F. Yin, K. Kido, and S. Tsurekawa, "Electron-beam-induced current study of grain boundaries in multicrystalline silicon," J. Appl. Phys., vol. 96, no. 10, pp. 5490-5495, Nov. 2004.

[12] G. Coletti, R. Kvande, V. D. Mihailetchi, L. J. Geerligs, L. Arnberg, and E. J. Ovrelid, "Effect of iron in silicon feedstock on p- and n-type multicrystalline silicon solar cells," J. Appl. Phys., vol. 104, no. 10, p. 104913, 2008.

[13] K. Adamczyk et al., "Recombination activity of grain boundaries in high-performance multicrystalline Si during solar cell processing," J. Appl. Phys., vol. 12, no. 5, p. 055705, 2018.

[14] D. Tweddle et al., "Atom Probe Tomography of Fast-Diffusing Impurities and the Effect of Gettering in Multicrystalline Silicon," in AIP Conference Proceedings, 2018, vol. 130019, p. 1999.

[15] J. Lindroos et al., "Nickel: A very fast diffuser in silicon," J. Appl. Phys., vol. 113, no. 20, 2013.

[16] M. K. Bakhadyrkhanov, S. Zainabidinov, and A. Khamidov, "Some characteristics of diffusion and electrotransport of nickel in silicon," Sov. Phys. Semicond., vol. 243, no. 14, 1980.

[17] S. K. Estreicher, D. J. Backlund, C. Carbogno, and M. Scheffler, "Activation energies for diffusion of defects in silicon: The role of the exchange-correlation functional," Angew. Chemie - Int. Ed., vol. 50, no. 43, pp. 10221-10225, 2011.

[18] M. Hourai et al., "A method of quantitative contamination with metallic impurities of the surface of a silicon wafer," Jpn. J. Appl. Phys., vol. 27, no. 12A, pp. L2361-L2363, 1988.

[19] J. Lindroos and H. Savin, "Review of light-induced degradation in crystalline silicon solar cells," Sol. Energy Mater. Sol. Cells, vol. 147, pp. 115-126, 2016.

[20] A. A. Istratov and E. R. Weber, "Electrical properties and recombination activity of copper, nickel and cobalt in silicon," Appl. Phys. A Mater. Sci. Process., vol. 66, no. 2, pp. 123-136, 1998.

[21] E. R. Weber, "Transition metals in silicon,” Appl. Phys. A Mater. Sci. Process., vol. 30, no. 1, pp. 1-22, 1983.

[22] G. Coletti, "Sensitivity of state-of-the-art and high efficiency crystalline silicon solar cells to metal impurities," Prog. Photovoltaics Res. Appl., vol. 21, pp. 1163-1170, Mar. 2013.

[23] N. Yarykin and J. Weber, "Evidence for room-temperature in-diffusion of nickel into silicon," Appl. Phys. Lett., vol. 109, no. 10, pp. 2014-2017, 2016.

[24] A. Stoffers, O. Cojocaru-Mirédin, W. Seifert, S. Zaefferer, S. Riepe, and D. Raabe, "Grain boundary segregation in multicrystalline silicon: correlative characterization by EBSD, EBIC, and atom probe tomography," Prog. Photovoltaics Res. Appl., vol. 23, no. 12, pp. 1742-1753, Dec. 2015. 
[25] Y. Ohno et al., "Recombination activity of nickel, copper, and oxygen atoms segregating at grain boundaries in mono-like silicon crystals," Appl. Phys. Lett., vol. 109, p. 142105, 2016.

[26] C. Lotharukpong et al., "Specimen preparation methods for elemental characterisation of grain boundaries and isolated dislocations in multicrystalline silicon using atom probe tomography," Mater. Charact., vol. 131, pp. 472-479, 2017.

[27] L. Wang, W. Yu, and S. Shen, "Revisiting the structures and energies of silicon $\langle 110\rangle$ symmetric tilt grain boundaries," J. Mater. Res., no. Md, pp. 1-13, 2019.

[28] V. Kveder, M. Kittler, and W. Schröter, "Recombination activity of contaminated dislocations in silicon: A model describing electron-beam-induced current contrast behavior," Phys. Rev. B, vol. 63, no. 11, p. 115208, 2001.

[29] M. Kittler and W. Seifert, "On the Origin of EBIC Defect Contrast in Silicon," Phys. Status Solidi, vol. 138, pp. 687-693, 1993.

[30] N. Yarykin and J. Weber, "Nickel in silicon: Room-temperature in-diffusion and interaction with radiation defects," Phys. Status Solidi Curr. Top. Solid State Phys., vol. 14, no. 7, p. $1700005,2017$.

[31] R. L. Meek, T. M. Buck, and C. F. Gibbon, "Silicon Surface Contamination: Polishing and Cleaning,” ECS J. Solid State Sci. Technol., vol. 120, no. 9, pp. 1241-1246, 1974.

[32] L. T. Canham, M. R. Dyball, and K. G. Barraclough, "Surface copper contamination of asreceived float-zone silicon wafers," J. Appl. Phys., vol. 66, no. 2, pp. 920-927, 1989.

[33] T. Buonassisi, A. A. Istratov, M. D. Pickett, M. A. Marcus, T. F. Ciszek, and E. R. Weber, "Metal precipitation at grain boundaries in silicon: Dependence on grain boundary character and dislocation decoration," Appl. Phys. Lett., vol. 89, no. 4, pp. 1-4, 2006.

[34] J. Chen and T. Sekiguchi, "Carrier recombination activity and structural properties of smallangle grain boundaries in multicrystalline silicon," Japanese J. Appl. Physics, Part 1 Regul. Pap. Short Notes Rev. Pap., vol. 46, no. 10 A, pp. 6489-6497, 2007.

[35] T. Sameshima et al., "Interaction between metal impurities and small-angle grain boundaries on recombination properties in multicrystalline silicon for solar cells," Appl. Phys. Express, vol. 5, no. 4, 2012.

[36] Z. Xi, D. Yang, J. Chen, and T. Sekiguchi, "Recombination behavior of nickel in cast multicrystalline silicon," Mater. Sci. Semicond. Process., vol. 9, no. 1-3, pp. 304-307, 2006.

[37] R. Rizk, X. Portier, G. Allais, and G. Nouet, "Electrical and structural studies of copper and nickel precipitates in a $\Sigma=25$ silicon bicrystal," J. Appl. Phys., vol. 76, no. 2, pp. 952-958, 1994.

[38] M. S. Wiig, K. Adamczyk, H. Haug, K. E. Ekstrøm, and R. Søndenå, "The Effect of Phosphorus Diffusion Gettering on Recombination at Grain Boundaries in HPMC-Silicon Wafers," Energy Procedia, vol. 92, no. 1876, pp. 886-895, 2016.

[39] T. S. Fell, P. R. Wilshaw, and M. D. De Coteau, "EBIC investigations of dislocations and their interactions with impurities in silicon," Phys. Status Solidi, vol. 138, no. 2, pp. 695-704, 1993. 


\section{Highlights}

- Correlative microscopy approach for the analysis of grain boundaries in silicon

- Colloidal silica polishing increases defect recombination activity

- APT confirms nickel and copper contamination from polishing

- Room temperature diffusion of nickel and copper occurs 